\title{
In vivo skin penetration and metabolic path of quantum dots
}

\author{
TANG Lei ${ }^{1}$, ZHANG ChunLing ${ }^{2 *}$, SONG GuangMing ${ }^{1}$, JIN Xun $^{1} \&$ XU ZhongWei ${ }^{1}$ \\ ${ }^{1}$ Logistics University of Chinese People's Armed Police Force, Tianjin 300162, China; \\ ${ }^{2}$ College of Physics Science, Nankai University, Tianjin 300071, China
}

Received November 5, 2011; accepted October 31, 2012; published online January 17, 2013

\begin{abstract}
The skin is the largest organ of the body and is a potential route of exposure to sunscreens and cosmetics containing nanoparticles; however, the permeability of the skin to these nanoparticles is currently unknown. In this paper, we studied the transdermal delivery capacity through mouse skin of water-soluble CdSeS quantum dots (QDs) and the deposition of these QDs in the body. QD solution was coated onto the dorsal hairless skin of male ICR mice. Fluorescence microscopy and transmission electron microscopy (TEM) were used to observe the distribution of QDs in the skin and organs, and inductively coupled plasma-mass spectrometry (ICP-MS) was used to measure the ${ }^{111} \mathrm{Cd}$ content to indicate the concentration of QDs in plasma and organs. Experimental results indicate that QDs can penetrate into the dermal layer and are limited to the uppermost stratum corneum layers and the hair follicles. Through blood circulation, QDs deposit mostly in liver and kidney and are difficult to clear. ${ }^{111} \mathrm{Cd}$ concentration was greater than $14 \mathrm{ng} \mathrm{g}^{-1}$ in kidney after $120 \mathrm{~h}$ after $0.32 \mathrm{nmol}$ QDs was applied to a mouse. These results suggest that QDs have in vivo transdermal delivery capacity through mouse skin and are harmful to the liver and kidney.
\end{abstract}

quantum dots, nanoparticles, skin penetration, metabolism, ICP-MS

Citation: Tang L, Zhang C L, Song G M, et al. In vivo skin penetration and metabolic path of quantum dots. Sci China Life Sci, 2013, 56: 181-188, doi: $10.1007 / \mathrm{s} 11427-012-4404-\mathrm{x}$

Nanoparticles are used in applications ranging from targeted fluorescent labels in life sciences [1], ultraviolet radiation (UVR) protective cosmetics [2], and bacterial inhibitors [3] in food storage containers to wound care products and baby pacifiers. However, these applications also raise environmental and human health concerns [4].

The skin is the largest organ of the body and is a potential route of exposure to engineered nanomaterials, such as sunscreens and cosmetics. Although skin is a remarkably efficient natural barrier, it is also a semi-permeable and nanoporous barrier. Nanomaterials have the possibility of migrating through this nanoporous membrane. Therefore, an understanding of the transdermal delivery of nanoparticles is important for nanomaterials risk assessment.

*Corresponding author (email: zhangchl@nankai.edu.cn)
Semiconductor quantum dots (QDs) are semiconductor nanocrystals with diameter between 2 and $100 \mathrm{~nm}$. They are generally prepared from group II and VI elements or group III and V elements. QDs have advanced optical properties compared with traditional organic fluorescent materials, such as high brightness, broad absorption and narrow emission spectra, tunable emission maxima and longer fluorescence lifetime. Therefore, the most promising applications of QDs are drug delivery, imaging markers, diagnostics or therapeutics [5]. However, many of the ions which can be released from QDs such as $\mathrm{Cd}^{2+}, \mathrm{Se}^{2-}$ and $\mathrm{Te}^{2-}$, are toxic to cells and animals. Some QDs have a core-shell structure and the shell has been shown to minimize Cd leaching [6]; however, the QD surface coatings and charge can influence the toxicity of QDs $[7,8]$. Thus it is important to pay close attention to their toxicity because the QDs are just several 
nanometers in diameter. Currently, the majority of toxicological studies on QDs focus on cellular toxicity, but the studies on in vivo skin penetration and metabolism are relatively lacking. For example, spherical QD565 and elliptical QD655 with three different surface coatings in flow-through diffusion cells showed penetration in porcine skin [9]. Carboxylated QDs applied to the skin of SKH-1 mice in a glycerol vehicle could penetrate intact and UVR compromised skin barrier [10]. QDs conjugated with diphtheria toxoid could be effectively delivered through mouse skin under hyperthermic conditions [11].

In this work, the transdermal delivery capacity of QDs through mice skin under normal conditions was investigated. Water-soluble QDs were directly coated onto the dorsal hairless skin of ICR mice. The distribution of QDs in skin was accurately calibrated, and the concentration of $\mathrm{Cd}$ in main organs and the toxicity of QDs to Human Epidermal Keratinocytes (HEK) cells were measured. The results of this study indicate that the skin is surprisingly permeable to QDs, which has important implications for a nanomaterials risk assessment.

\section{Materials and methods}

\subsection{Quantum dots}

Quantum dots used in this study were purchased from Tianjin Yourui Quantum Dots Co. Ltd. The QDs have a core of $\mathrm{CdSeS}$ and a shell comprised of a silica network with multiple hydroxyls to promote solubility in water. The QDs have negative charge. Their average size was analyzed by transmission electron microscope (TEM). Their diameter is about $20 \mathrm{~nm}$, as shown in Figure 1.

The concentration of the QD solution was adjusted to 4 $\mu \mathrm{mol} \mathrm{L}^{-1}$ and the $\mathrm{pH}$ value was about 8.0. Before use in animal experiments, the QD solutions were stored in the refrigerator with opaque glass to prevent light decomposition. To ensure the dispersion of QDs, the QD solution was first sonicated for $2 \mathrm{~min}$ with an ultrasonic oscillator. To test the stability of the QDs fluorescence, $40 \mu \mathrm{L}$ of QD so-

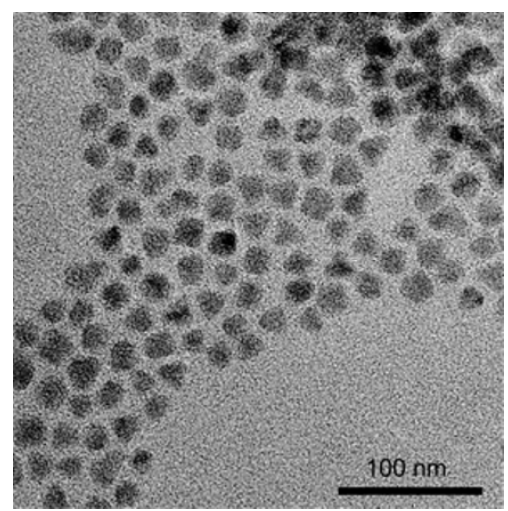

Figure 1 TEM image of CdSeS quantum dots. Their diameter is about $20 \mathrm{~nm}$. lution was dropped onto a glass slide and placed on animal feeding shelves. Fluorescence images of the same position were taken with the same exposure time for seven consecutive days. We also tested the quantitative characterization of the fluorescence peak and intensity of QDs with a fluorescence spectrometer (F-4000, Hitachi, Japan). First, the fluorescence spectroscopy was measured immediately after the QD solution was taken out of the refrigerator and sonicated. Then the QD solution was stored in a translucent glass botthe in the animal breeding room. Eighty hours later, the fluorescence spectroscopy was measured again.

The fluorescence spectrum indicates that their peak wavelength is about $620 \mathrm{~nm}$. There are two reasons for why we used silica-coated CdSeS rather than other types of QDs. First, we want to study the distribution of QDs on mice skin. The emission wavelength of CdSeS QDs is about $620 \mathrm{~nm}$, so their emission light can be observed under a fluorescent lamp. Therefore, we can easily judge the distribution of QDs on mice skin by observing their fluorescence intensity and distribution under a fluorescent lamp. Second, $\mathrm{Cd}$ is toxic to living organisms, but the shell of silica can largely reduce its toxicity.

\subsection{Animals}

All of the animal experiments were performed in compliance with the local ethics committee. Male ICR mice $((25 \pm 1) \mathrm{g}$, provided by the laboratory animal center of the Academy of Military Medical Sciences) were used as the experimental model because of their low cost and strong vitality compared with nude mice. Moreover, mouse skin is usually used as a drug delivery model membrane. Mice were anesthetized by pentobarbital. The hair of the dorsal skin was carefully cut with surgical scissors, and then the shaved part of the skin was wiped with warm water. Cosmetic grade hair removal cream was applied for 3 min and then was removed with warm water. The skin was allowed to dry naturally to complete the hair removal work. Each area of exposed skin was about $1.5 \mathrm{~cm}^{2}$. Optical microscope was used to observe whether the skin was damaged. Mice without injury were kept in sterile cages in ventilated animal rooms.

\subsection{QDs dose}

Twenty-four hours after hair removal, mice were anesthetized again with pentobarbital. Approximately $80 \mu \mathrm{L}$ of the QD solution (containing $0.32 \mathrm{nmol}$ QDs) was carefully and evenly coated onto the dorsal hairless skin of each mouse and was allowed to dry naturally. To prevent friction between the parts coated with QDs after the mice regained consciousness, each mouse was housed in a separate feeding cage. Six mice without QDs were used as a negative control group. 


\subsection{Plasma and organs collection}

Time intervals of 1, 6, 12, 24, 48 and $120 \mathrm{~h}$ after QDs coating were used. Fluorescent images of the mice were taken under fluorescent light (wavelength $352 \mathrm{~nm}$ ) at each time interval and then the mice were killed by cervical dislocation. Blood samples (about $0.8-1.0 \mathrm{~mL}$ for each mouse) retrieved from the ocular vein were mixed and placed in heparinized-anticoagulated tubes. Skin coated with QDs and the hearts, livers, spleens, lungs and kidneys were collected immediately. Half of the skin and the organs were placed in an embedding medium, which was pre-dropped on ice, to make tissue slices. The other half of the skin was placed in Trump's fixative for TEM and the other half of the organs were used for QDs concentration analysis. The mice of the control group were also killed, and their plasma and organs were collected.

\subsection{Fluorescent images}

To assess the localization of QDs in the skin, heart, liver, spleen, lung and kidney, the skin and organ samples collected at each time intervals as well as the controls were subjected to pathological examination. The skin and the organs were sectioned in a cryostat into slices of $10 \mu \mathrm{m}$ thickness. To prevent QDs contamination between slices, the blade was wiped with clean silk, and skin samples were cut perpendicular to the knife with the skin standing upright so that sectioning was conducted from the bottom of the dermis up through the epidermal layers. To observe tissue structure with an optical microscope, odd-numbered slices were Hematoxylin-Eosin (HE) stained. To prevent weakening of the QDs fluorescence, even-numbered slices were placed on glass slides without dye and without mounting medium, and were fixed only with acetone. To accurately determine the distribution of QDs on skin, skin slices from 1 and $6 \mathrm{~h}$ were pre-dyed with 4',6-diamidino-2-phenylindole (DAPI) before fluorescence observation. The bright-field and corresponding fluorescent images of the slices were observed by inverted fluorescent microscope.

\subsection{TEM of skin sections}

The dorsal hairless skin was cut off $6 \mathrm{~h}$ after being coated with QDs solution. The skin sample was pre-fixed in Trump's at $4{ }^{\circ} \mathrm{C}$ for $24 \mathrm{~h}$ and then successively fixed in $2.5 \%$ glutaraldehyde and $1 \%$ osmium tetroxide, dehydrated through graded ethanol solutions, intergraded in propylene oxide, and embedded in Epon812. Unstained sections $(50 \mathrm{~nm})$ were mounted on copper grids and then examined on the TEM. Furthermore, mice without QDs were used as a negative control group and the TEM images of the control group were also taken.

\subsection{QD concentration analysis}

QD analysis was based on the quantification of ${ }^{111} \mathrm{Cd}$ in various samples by ICP-MS. The plasma in heparinizedanticoagulated tubes were centrifuged at $2000 \mathrm{r} \mathrm{min}^{-1}$ for $10 \mathrm{~min}$ and the upper clear solution was withdrawn. The organs were individually weighed and immersed in $1 \mathrm{~mL}$ of distilled water in a glass grinder to ground into a mash. To make the ICP-MS sample, the upper clear solution of plasma and the organ homogenates were digested by the addition of a 4:1 mixture of nitric acid and perchloric acid. The final solutions were injected into the ICP-MS system to determine the concentration of cadmium.

\subsection{Cell culture and viability assay}

Neonatal HEK were seeded in 96-well culture plates at approximately 7000 cells per well and grown in a humidified environment of $5 \% \mathrm{CO}_{2}$ at $37^{\circ} \mathrm{C}$ with keratinocyte growth media (KGM-2) cell medium. Upon reaching $70 \%$ confluency, the cells were exposed to QDs in KGM-2. The control wells consisted of only KGM-2 and cells. The cells were treated with QD solutions with concentrations of 40 (equal to $800 \mathrm{nmol} \mathrm{L}{ }^{-1}$ ), $4,0.4,0.04,0.004$ and $0.0004 \mu \mathrm{g} \mathrm{mL}^{-1}$. HEK at 24, 48 and $72 \mathrm{~h}$ were assayed using MTT (3-(4,5-dimethylthiazol-2-yl)-2,5-diphenyltetrazolium bromide) for viability as described by Mosmann [12]. The absorbance, directly proportional to cell viability, was determined spectrophotometrically at $490 \mathrm{~nm}$ in a plate reader. The results from this study provided the range of QD concentrations that were not cytotoxic to HEK.

\section{Results}

\subsection{Quantum dots fluorescence}

Fluorescence images of QDs on a glass slide showed that the fluorescence intensity of the adjacent two days had no significant difference and there was a slight difference between the $6 \mathrm{~h}$ and 7 days fluorescence images, as shown in Figure $2 \mathrm{~A}$ and B. Figure $3 \mathrm{~A}$ and $\mathrm{B}$ shows the fluorescence spectroscopy of QD solutions immediately after being taken out of the refrigerator and $80 \mathrm{~h}$ after being stored in a translucent glass bottle in the animal breeding room. In Figure $3 \mathrm{~A}$, the fluorescence peak is $621.50 \mathrm{~nm}$, the peak intensity is 54.6 and the FWHM is $34.2 \mathrm{~nm}$. In Figure 3B, the fluorescence peak is $620.60 \mathrm{~nm}$, the peak intensity is 54.1 and the FWHM is $35.4 \mathrm{~nm}$. The fluorescence peak, the peak value and the FWHM in the two spectroscopy measurements is essentially the same. Therefore, it can be concluded that the QDs are fluorescently stable under experimental conditions.

\subsection{Skin surface fluorescence}

After natural drying, mouse skin coated with QDs has the 

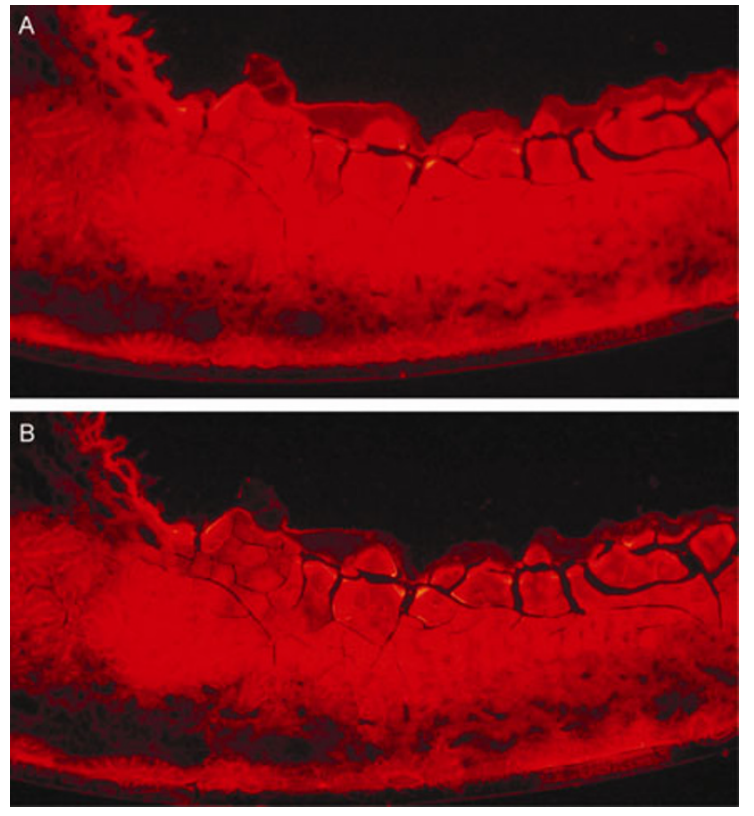

Figure 2 Fluorescence images of a glass slide $6 \mathrm{~h}(\mathrm{~A})$ and $7 \mathrm{~d}(\mathrm{~B})$ after coating with QD solutions. The fluorescence images were taken with the same exposure time.

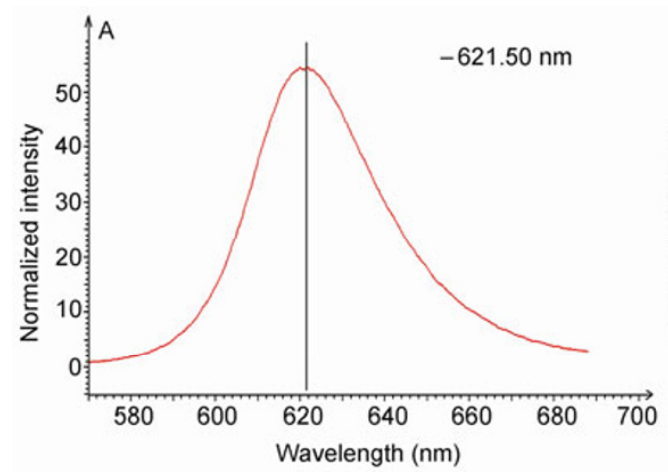

same color as that of skin without QDs in natural light, but shows bright red fluorescence under fluorescent light. In Figure $4 \mathrm{~A}-\mathrm{C}$ are the fluorescent images of three mice 12 , 48 and $120 \mathrm{~h}$ after coating with QD solutions. The fluorescence in Figure 4A is similar to that in Figure 4B, but brighter than that in Figure 4C. Overall, QDs appear red fluorescent for a period of time, but the fluorescence faded slowly with time. As the fluorescent intensity of QDs on the glass slide is relatively stable, the fade of the fluorescence intensity on the skin may be caused by the following facts: (i) some QDs have migrated into the body of the mouse, (ii) the fluorescence is masked by the bright blue fluorescence emitted by renascent hair, (iii) some QDs may be removed by friction between the mice and the animal bedding (sawdust). The last reason may be minor because the QDs were coated on the dorsal skin and the chance of QDs touching the bedding was small [13].

\subsection{Optical microscopy of skin}

After HE staining, odd-numbered skin slices were observed under the optical microscope. Figure $5 \mathrm{~A}$ and $\mathrm{B}$ shows the

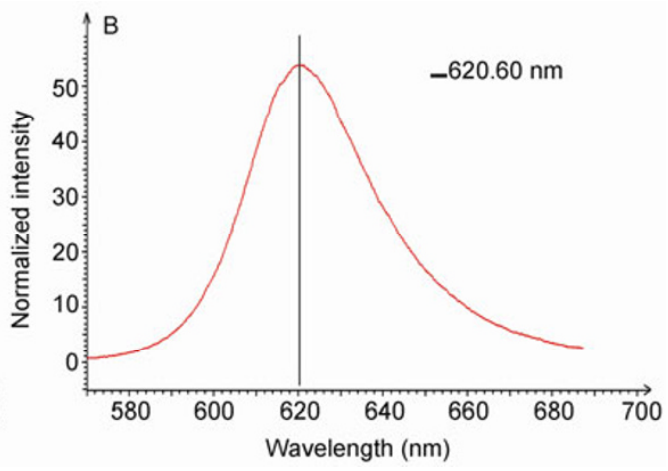

Figure 3 A and B are the fluorescence spectroscopy of QD solutions immediately after being taken out of the refrigerator and $80 \mathrm{~h}$ after being stored in a translucent glass bottle in the animal breeding room.
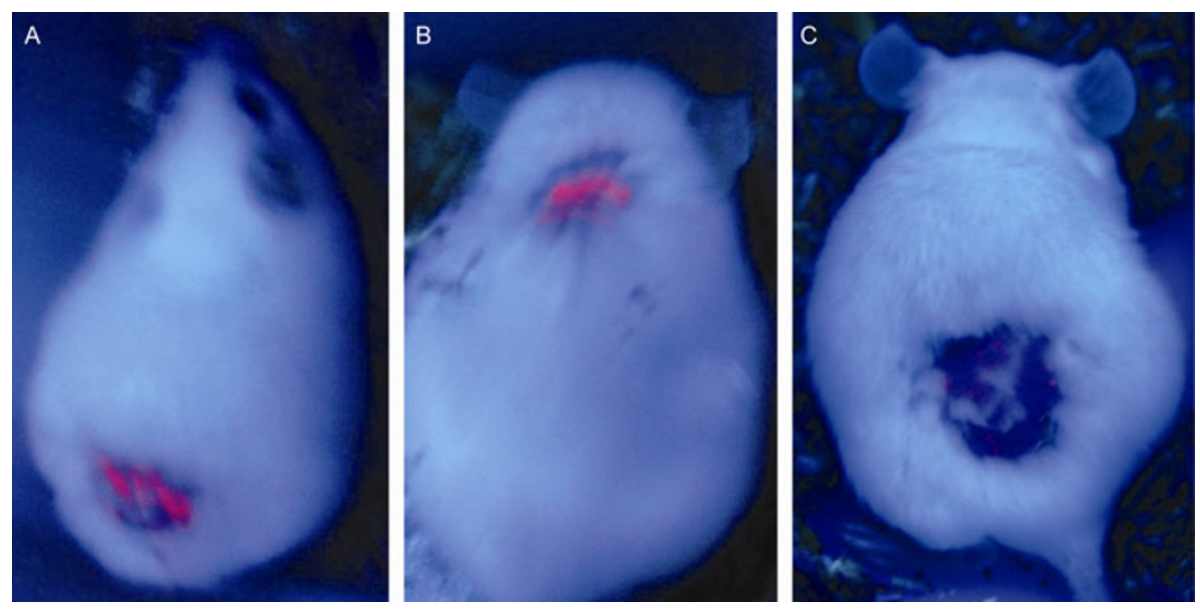

Figure 4 Fluorescence images of mice 12 (A), 48 (B) and $120 \mathrm{~h} \mathrm{(C)} \mathrm{after} \mathrm{coating} \mathrm{with} \mathrm{QD} \mathrm{solutions.} \mathrm{The} \mathrm{dorsal} \mathrm{hairless} \mathrm{skin} \mathrm{of} \mathrm{each} \mathrm{mouse} \mathrm{was} \mathrm{coated}$ with $80 \mu \mathrm{L}$ of the QD solution (containing $0.32 \mathrm{nmol}$ QDs). 
optical microscopy of skin slices 1 and $6 \mathrm{~h}$ after coating with QD solution.

In Figure 5A, there are some sweat glands, including the secretion sites (1) and the catheters (2). In Figure 5B, epidermis (E), dermis (D) and the fat particles (F) are visible. Cross-sections of hair follicles $(\mathrm{H})$ are obvious. Sebaceous glands $(\mathrm{S})$ are located on both sides of the hair follicle. Because the mouse skin is thinner, the thickness of the stratum corneum cannot be clearly measured.

\subsection{Fluorescence microscopy of skin}

Even-numbered skin slices and organ slices were observed by inverted fluorescence microscopy to assess the localization of the QDs. Figure 6A and B is the fluorescence images of skin 1 and $6 \mathrm{~h}$ after coating with QD solution. The blue dots indicate the position of the nuclei and the red dots indicate the position of the QDs. By studying the skin structure in Figure 5 and contrasting with the fluorescence image in Figure 6, we could determine that most QDs gather on the skin surface, and a small amount gathers in the hair follicles. Because hair follicles have openings on the skin surface, it is easy for nano-size QDs to gain access through hair follicle openings and accumulate in the hair follicle. This is similar to Alvarez-Roman's [14] observation that nanoparticles aggregated in hair follicle openings.

Figure $6 \mathrm{C}$ and D is magnified sections of Figure $6 \mathrm{~A}$ and B. Upon careful observation, we can see there are more QDs in Figure 6D between the nucleus under the skin surface than there are in Figure 6C. These results show that QDs can slowly penetrate the epidermis of the intact skin.

We did not observe single QDs in the skin and organs slices. This may be because QDs are nanoscale and the general fluorescence microscopy cannot achieve nanoscale resolution. However, in theory, there must exist QDs in the skin and organs. This is because the hydrophilic nanopore density in the stratum corneum of the skin is taken to be $10^{8}-10^{9} \mathrm{~cm}^{-2}$ [15] and the radius of hydrophilic pores of hairless mouse skin is $2.7 \mathrm{~nm}$. The pore width of the ICR mouse must be large enough for QDs used in this study to
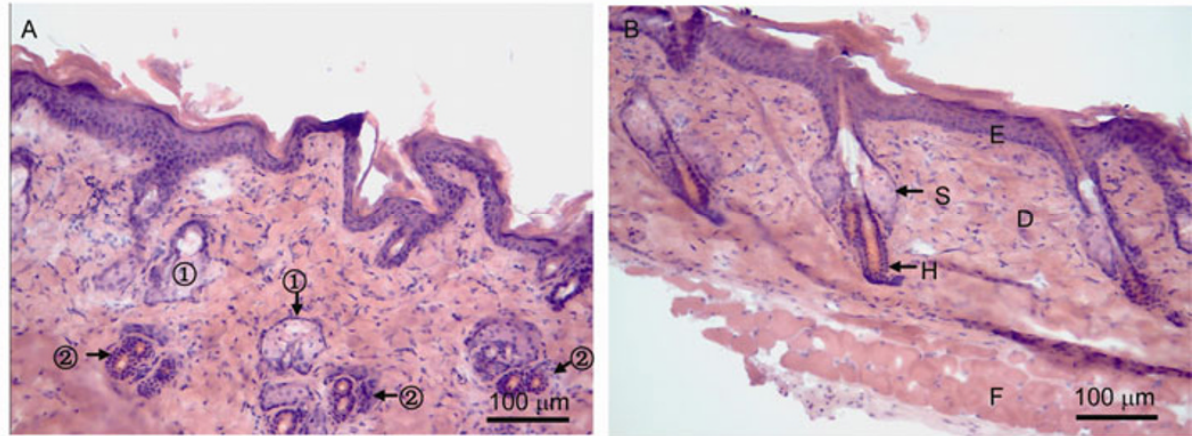

Figure 5 Optical microscopy of skin slices after HE staining. A and B, 1 and $6 \mathrm{~h}$ after coating with QD solutions. Note the secretion sites (1) and catheters (2) of sweat glands in Figure A and the epidermis (E), dermis (D), fat particles (F), hair follicles (H) and sebaceous glands(S) in Figure B.
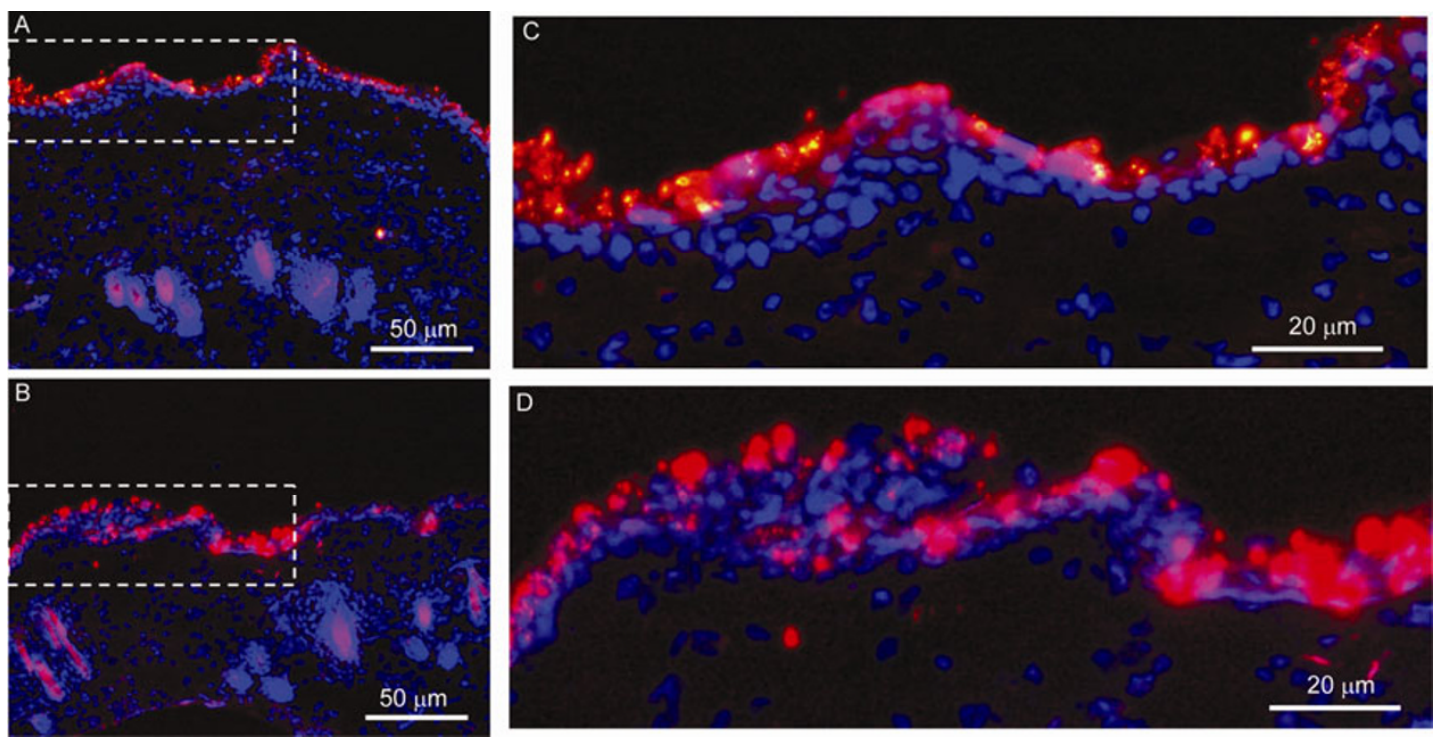

Figure 6 The fluorescence images of skin after DAPI dyeing. A and B, 1 and $6 \mathrm{~h}$ after coating with QDs solution. C and D, A higher magnification of the enlarged area in A and B. 
undergo permeation, so QDs can penetrate through skin and then migrate into the main organs through blood circulation.

\subsection{TEM of QDs in skin}

TEM was used to assess the precise location and distribution of QDs in the skin. Figure 7A is the TEM image of the control group. There are distinct spaces between stratum corneum (SC) cells, but there are no QDs in each layer. Figure $7 \mathrm{~B}$ and $\mathrm{C}$ is the TEM images of the skin $6 \mathrm{~h}$ after being coated with QDs solution. In Figure 7B, the QDs are localized within the uppermost layers of the SC and adhere to the two sides of the SC cell as agglomerates or as line-like structures. Greater amounts of QDs were found in the superficial layers of the SC with a decrease in QDs concentration deeper into the SC layers. Higher magnification of the enlarged area in Figure 7B shows individual QDs and some small agglomerates (Figure 7C). The QDs used in this study are similar in shape, size and electron density to ribosomes in cells. We cannot identify if the dots in the circle in Figure 7B are QDs or ribosomes. If nanoparticles with a special shape or larger electron density were used in the experiment, such as nail-shaped QDs or colloidal silver, the dots in the cells could be easier to identify.

\subsection{Cd concentration}

The QDs distributed in different organ tissues were also detected by the ICP-MS analysis of ${ }^{111} \mathrm{Cd}$ (Figure 8). The peak concentration of QDs in the liver and kidney occurred $12 \mathrm{~h}$ after skin coating. There were over $10 \mathrm{ng} \mathrm{g}^{-1}$, twice their control group. The $7 \mathrm{ng} \mathrm{g}^{-1}$ peak concentration in the spleen was achieved $6 \mathrm{~h}$ after skin coating.

Because the ICP-MS instrument detection accuracy is relatively high, little cadmium can also be detected. We detected a certain amount of cadmium content in the control group. This cadmium may come from the diet or environmental exposure. Because the mice used in this study belong to the same company and the same batch, all the mice should have the same background cadmium content. So, we determined that the increased cadmium content in the studied organs was indeed caused by the QDs.

We conclude that nanometer-sized QDs can permeate through skin and may further migrate into the main organs of mice. The metabolic rate of QDs in various organs is different. The QDs accumulated in the liver and kidney cannot be effectively eliminated, but the QDs in the spleen could be thoroughly eliminated within $24 \mathrm{~h}$.

In this work, cadmium concentration in the liver, spleen and kidney increased slowly and then decreases slowly with time, which is different with Chen et al's article [16], in which the cadmium concentration in the organs increased rapidly and then decreased rapidly. We believe that this is because Zhen Chen and colleagues injected QD solutions through the tail vein, resulting in a cadmium concentration in blood that increased rapidly after injection. However, in our study, QD solutions applied to the skin of mice pene-
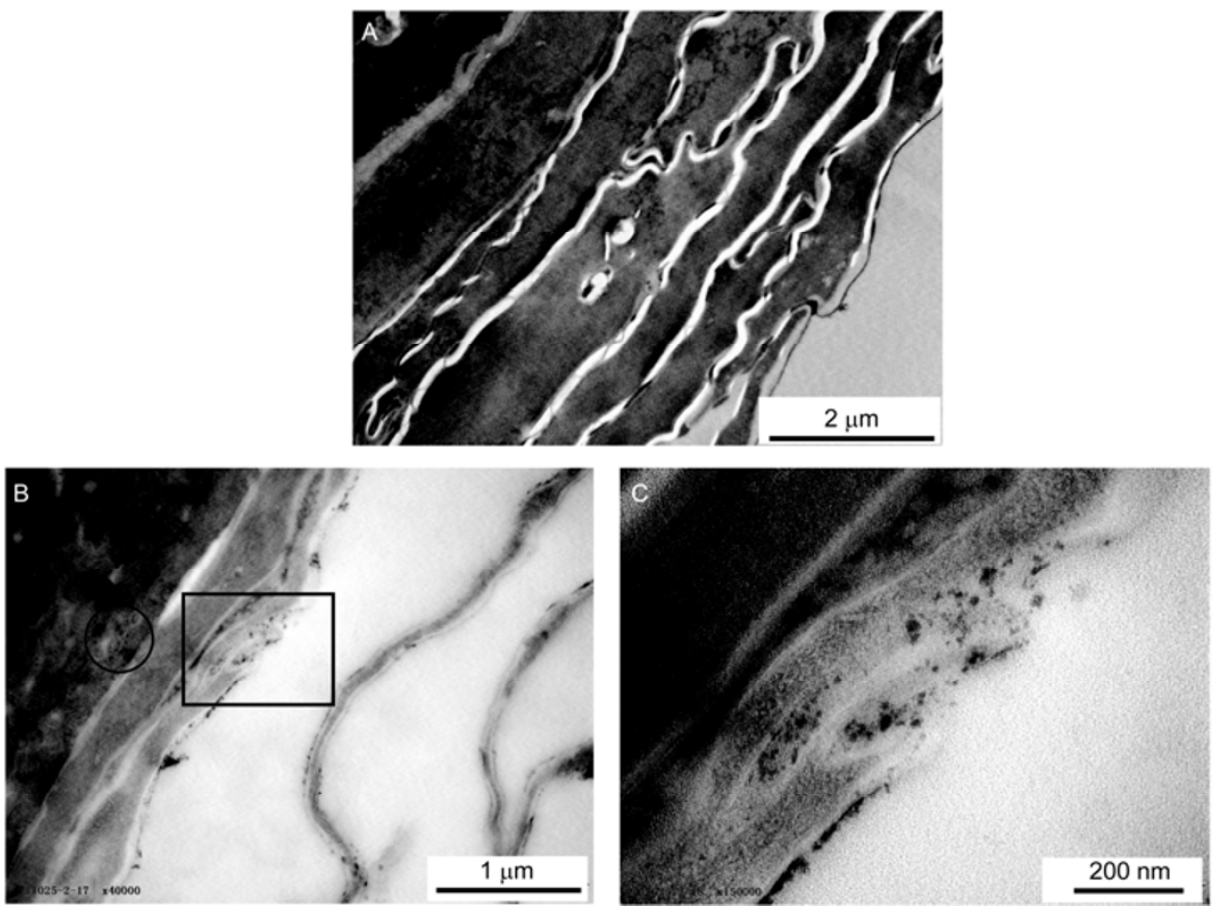

Figure 7 TEM images of the stratum corneum of the control group (A) and that $6 \mathrm{~h}$ after being coated with QDs solution (B and C). A, There are distinct spaces between stratum corneum (SC) cells, but there is no QDs in each layer. B, QDs adhere to the two sides of the stratum corneum cell. C, Higher magnification of the enlarged area in B showing individual QDs and some small agglomerates. 
trated slowly, and sweat and sebum secreted on the skin will promote the further penetration of QDs even if the solution has dried naturally. Therefore, the method of coating QD solutions is a relatively slow process.

\subsection{MTT viability}

The MTT assay was used to assess the viability of HEK treated with 40 (equal to $800 \mathrm{nmol} \mathrm{L}^{-1}$ ), 4, 0.4, 0.04, 0.004 and $0.0004 \mu \mathrm{g} \mathrm{mL}^{-1}$ of QDs. There was a statistically significant decrease in viability at $40(* *, P<0.1)$ and $4 \mu \mathrm{g}$ $\mathrm{mL}^{-1}(*, P<0.05)$ at 24,48 and $72 \mathrm{~h}$, which was dose dependent (Figure 9). The 0.4, 0.04, 0.004 and $0.0004 \mu \mathrm{g}$ $\mathrm{mL}^{-1}$ concentrations of QDs did not show a significant decrease in viability at 24,48 and $72 \mathrm{~h}$.

\section{Discussion}

The skin is the largest organ of the body and is a natural barrier to outside pollutants. However, the toxicity of QDs in skin needs to be investigated since the QDs are nanometers in diameter and exposure could occur during the production process and in consumers during biological or medical applications. The penetration, localization, and toxicity of QDs in skin and skin cells have become a critical issue as the uses for QDs increases.

In this work, silica-coated CdSeS QDs with a hydrody-

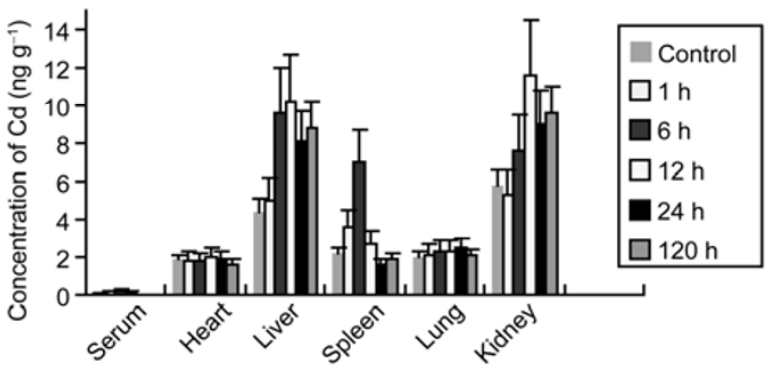

Figure 8 Concentration of $\mathrm{Cd}$ in main organs at different time intervals (data are represented as means, $n=6$ for each value).

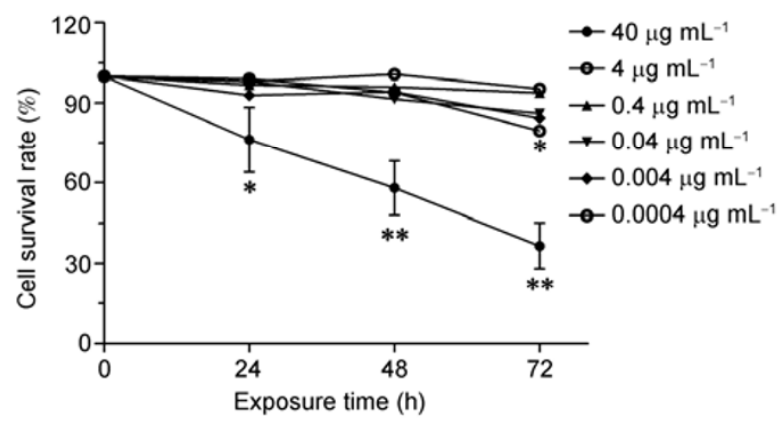

Figure 9 MTT cell survival rate of HEK treated with QDs. Mean viability at 24,48 and $72 \mathrm{~h}$. **, $P<0.1 ; *, P<0.05$. namic diameter of $20 \mathrm{~nm}$ were coated on the dorsal hairless skin of ICR mice. Fluorescence microscope images indicate that QDs were localized in the uppermost layers of the SC and the hair follicles. This is similar to other reports that found that QD621 were localized in the outer root sheath of porcine hair follicles [9], $\mathrm{TiO}_{2}$ microparticles and polystyrene nanoparticles distributed near orifices in human hair follicles [1,17], and metallic nanoparticles less than $10 \mathrm{~nm}$ were capable of penetrating the SC and hair follicles [18]. QDs can work their way between the corneocytes of the stratum corneum and penetrate deep in the epidermis and dermis of an in vivo model with UVR penetration exacerbation [10].

In this work, TEM indicate that greater amounts of QDs were found within the intercellular spaces of the outermost SC layers as depicted in Figure 7B. Higher magnification of this area showed individual QDs (Figure 7C) but often they appeared as agglomerates. The main possible mechanism for the permeation of QDs through mouse skin is that silica-coated CdSeS QDs are hydrophilic nanoparticles, which cross the skin mainly through hydrophilic pathways such as hair follicles, sweat pores, the intercellular spaces between the corneocytes and other nanopores.

Similar to other nanoparticles, QDs can penetrate the body's normal protective role of phagocytic cells, and enter the circulatory system and central nervous system through different mechanisms, such as through the respiratory tract epithelial cells directly into the blood circulation system, or distributed to each part of body through the lymphatic system and eventually excreted through the circulatory system [19-21]. However, QDs with different core or surface modifications may have a different body distribution and clearance methods [22]. ICP-MS results indicate that QDs used in this study can deposit in the organs through blood circulation. The concentration of QDs in the liver and kidney $120 \mathrm{~h}$ after coating with QDs was twice the control group. ${ }^{111} \mathrm{Cd}$ concentration is still greater than $14 \mathrm{ng} \mathrm{g}^{-1}$ in the kidney after $120 \mathrm{~h}$ if $0.32 \mathrm{~nm}$ QDs was previously applied on a mouse.

In summary, this study provides information on QDs penetration and distribution in skin and toxicity in organs. Penetration of QDs into the skin is limited to the uppermost SC layers and areas near hair follicles. QDs deposited heavily in the liver and kidney, and they were difficult to clear. Additional tests such as tape stripping or abrasion should be conducted to determine if penetration to this barrier would allow an enhancement of the absorption of QDs. Also, if QDs are available for use in biological applications, the lowest concentration of QDs with low toxicity showing high fluorescence intensity would be optimal for use.

The authors thank Prof. Zhao Yan of Nankai University for conducting the ICP-MS analysis. This work was supported by the Medical College of Chinese People's Armed Police Force in 2010 (WYZ201003). 
1 Gao X H, Cui Y Y, Levenson R M, et al. In vivo cancer targeting and imaging with semiconductor quantum dots. Nat Biotechnol, 2004, 22 : 969-976

2 Serpone N, Emeline A V. Modelling heterogeneous photocatalysis by metal-oxide nanostructured semiconductor and insulator materials: factors that affect the activity and selectivity of photocatalysts. Res Chem Intermed, 2005, 31: 391-432

3 Chen X, Schluesener H J. Nanosliver: a nanoproduct in medical application. Toxicol Lett, 2008, 176: 1-12

4 Hardman R. A toxicologic review of quantum dots: toxicity depends on physicochemical and environmental factors. Environ Health, 2006,114: 165-170

5 Michalet X, Pinaud F F, Bentolila L A, et al. Quantum dots for live cells, in vivo imaging, and diagnostics. Science, 2005, 307: 538-544

6 Cho S J, Maysinger D, Jain M, et al. Long-term exposure to CdTe quantum dots causes functional impairments in live cells. Langmuir, 2007, 23: 1974-1980

7 Hoshino A, Fujioka K, Oku T, et al. Physicochemical properties and cellular toxicity of nanocrystal quantum dots depend on their surface modification. Nano Lett, 2004, 4: 2163-2169

8 Kirchner C, Liedl T, Kudera S, et al. Cytotoxicity of colloidal CdSe and CdSe/ZnS nanoparticles. Nano Lett, 2005, 5: 331-338

9 Leshuai W Z, William W Y, Vicki L C, et al. Biological interactions of quantum dot nanoparticles in skin and in human epidermal keratinocytes. Toxicol Appl Pharmacol, 2008, 228: 200-211

10 Luke J M, Gunter O, Alice $\mathrm{P} P$, et al. In vivo skin penetration of quantum dot nanoparticles in the murine model: the effect of UVR. Nano Lett, 2008, 8: 2779-2787

11 Upadhyay P. Enhanced transdermal-immunization with diptheria-toxoid using local hyperthermia. Vaccine, 2006, 24: 5593-5598

12 Mosmann T. Rapid colorimetric assay for cellular growth and surviv- al: application to proliferation and cytotoxicity assays. J Immunol Methods, 1983, 65: 55-63

13 Chu M Q, Wu Q, Wang J, et al. In vitro and in vivo transdermal delivery capacity of quantum dots through mouse skin. Nanotechnology, 2007, 18: 455103-455108

14 Alvarez-Roman R, Naik A, Kalia Y N, et al. Skin penetration and distribution of polymeric nanoparticles. J Contr Release, 2004, 99: 53-62

15 Kohli A K, Alpar H O. Potential use of nanoparticles for transcutaneous vaccine delivery: effect of particle size and charge. Int J Pharm, 2004, 275: 13-17

16 Chen $\mathrm{Z}$, Chen $\mathrm{H}$, Meng $\mathrm{H}$, et al. Bio-distribution and metabolic paths of silica coated CdSeS quantum dots. Toxicol Appl Pharmacol, 2008, 230: 364-371

17 Lademann J, Weigmann H, Rickmeyer C, et al. Penetration of titanium dioxide microparticles in a sunscreen formulation into the horny layer and the follicular orifice. Skin Pharmacol Appl Skin Physiol, 1999, 12: 247-256

18 Baroli B, Ennas M G, Loffredo F, et al. Penetration of metallic nanoparticles in human full-thickness skin. J Invest Dermatol, 2007, 127: 1701-1712

19 Nemmar A, Hoet P H M, Vanqtdckeuborne B, et al. Passage of inhaled particles into the blood circulation in humans. Circulation, 2002, 105: 411-414

20 Oberdörster E. Manufactured nanomaterials (fullerenes, $\mathrm{C}_{60}$ ) induce oxidative stress in the brain of juvenile largemouth bass. Environ Health Persp, 2004, 112: 1058-1062

21 Oberdörster G, Sharp Z, Atudorei V, et al. Translocation of inhaled ultrafine particles to the brain. Inhalat Toxicol, 2004, 16: 437-445

22 Fischer H C, Liu L, Pang K S, et al. Pharmacokinetics of nanoscale quantum dots: in vivo distribution, sequestration, and clearance in the rat. Adv Funct Mater, 2006, 16: 1299-1305

Open Access This article is distributed under the terms of the Creative Commons Attribution License which permits any use, distribution, and reproduction in any medium, provided the original author(s) and source are credited. 